\title{
Monocyte HLA-DR expression and neutrophil CD64 expression as biomarkers of infection in critically ill neonates and infants
}

\author{
Justin E. Juskewitch ${ }^{1}$, Roshini S. Abraham², Stacy C. League'2, Sarah M. Jenkins ${ }^{3}$, Carin Y. Smith ${ }^{3}$, Felicity T. Enders ${ }^{3}$, \\ Stefan K. Grebe ${ }^{2}$, William A. Carey ${ }^{4}$ and W. Charles Huskins ${ }^{5}$
}

\begin{abstract}
BACKGROUND: Reduced monocyte HLA-DR expression and increased neutrophil CD64 expression have been proposed as biomarkers of infection.
\end{abstract}

METHODS: From 2009-2011, blood samples from neonatal intensive care unit (NICU) and pediatric intensive care unit (ICU) patients $<1$ y of age were collected at enrollment and during subsequent evaluation for suspected infection, if it occurred. Samples were analyzed for monocyte HLA-DR and neutrophil CD64 expression levels by flow cytometry.

RESULTS: Forty-seven infants had study samples collected at enrollment; 26 infants had study samples collected at the time of a suspected infection. At enrollment, there was an inverse relationship between neutrophil CD64 expression and age ( $P \leq$ 0.047). At the time of suspected infection, infants with an infection demonstrated a lower percentage of HLA-DR+ monocytes $(P=0.02$, area under the curve (AUC) 0.78), higher percentage of CD64+ neutrophils ( $P=0.009$, AUC 0.81 ), and higher neutrophil CD64 expression levels ( $P=0.04$, AUC 0.75).

CONCLUSION: Monocyte HLA-DR and neutrophil CD64 expression in critically ill infants are related to age and infection.

I: nfection is a leading cause of morbidity and mortality in neonates and children in the United States $(1,2)$. In critically ill infants, the diagnosis of an invasive bacterial or fungal infection depends largely on blood cultures. However, the diagnostic utility of blood cultures is compromised by $24-48 \mathrm{~h}$ incubation times and reduced sensitivity due to low sample volumes and, in neonates, use of intrapartum antibiotics $(3,4)$. Standard biomarkers of inflammation, such as the total leukocyte count, neutrophil count, and acute phase reactant levels (C-reactive protein (CRP), procalcitonin (PCT)), have reasonable negative predictive value for infection, but their positive predictive value is limited (4-9).

Leukocyte surface molecules (e.g., monocyte HLA-DR, neutrophil CD64) have shown potential as new biomarkers of infection in this population. Increased CD64 expression is a marker of neutrophil activation in response to infection (1020). Decreased HLA-DR expression on monocytes is a measure of immune system "anergy" also believed to be a marker of infection $(21,22)$. Because critically ill infants are at higher risk for infection, it is important to examine the levels and kinetics of these molecules in the context of pre-existing critical illness (e.g., extreme prematurity, respiratory or circulatory failure) to better evaluate their utility as biomarkers of infection.

To that end, we developed a novel research infrastructure for real-time collection and prompt processing of blood samples for these labile cell surface molecules (23). The objective of this study was to examine monocyte HLA-DR and neutrophil CD64 expression in relation to postnatal age and infection in critically ill infants.

\section{RESULTS}

Patient Demographics

Between 1 April 2009 and 30 April 2011, 938 patients $<1$ y of age were admitted to the NICU and/or pediatric ICU of the Mayo Clinic Children's Center. Of these patients, 453 were eligible for the study and 146 were enrolled. Of all 146 enrolled subjects, $75 \%$ were less than $<7 \mathrm{~d}, 18 \%$ were $7-29 \mathrm{~d}$, and $7 \%$ were 1-11 mo of age.

\section{Enrollment Biomarker Levels in NonInfected Subjects}

Fifty-six subjects had enrollment samples collected (Figure 1a). The goal of this analysis was to assess the role of age on biomarker expression independent of infection. Nine of the subjects with enrollment samples were excluded since they were determined to have an infection at the time of enrollment leaving 47 noninfected subjects for analysis (Table 1 ). The majority of subjects in the two older age groups had delivered prematurely ( $<36$ wk gestational age).

We initially assessed monocyte, neutrophil, and lymphocyte counts in our enrollment samples (Supplementary Figure S1 online). Only neutrophil counts were different across age groups $(P=0.005)$ with lower counts in preterm vs. term neonates $<7 \mathrm{~d}$ of age ( $P=0.001$; Supplementary Figure S1b online).

\section{Monocyte HLA-DR Expression}

At enrollment, there was no significant difference across age groups in the percentage of HLA-DR+ monocytes (Figure 2a). The levels of monocyte HLA-DR expression, measured by the 
a

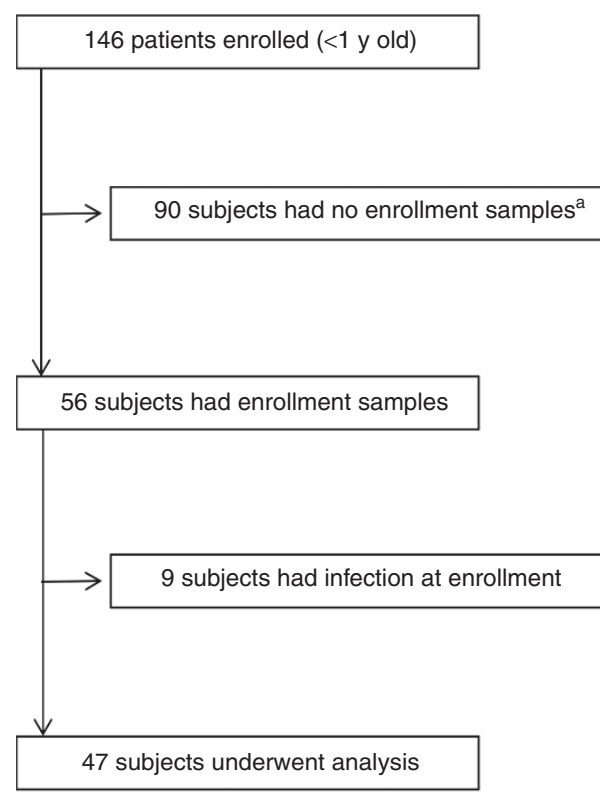

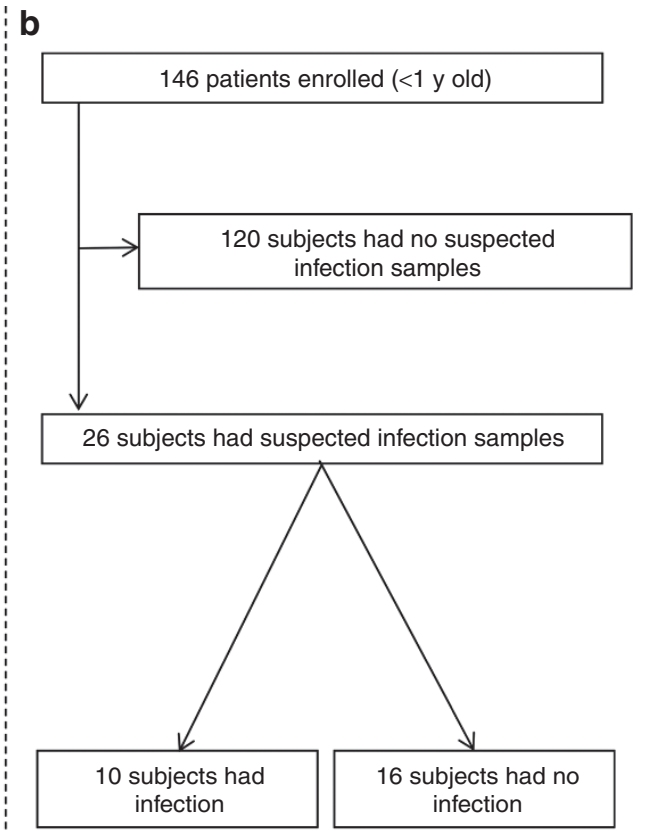

Figure 1. Overall study design. Study subject flow diagram for enrollment sample collection (a) and suspected infection sample collection (b). ${ }^{\mathrm{a} E n r o l l e d}$ subjects did not have an enrollment sample collected for the following reasons: (i) there were no clinically indicated blood tests within $72 \mathrm{~h}$ of enrollment; (ii) additional blood beyond that needed for clinically indicated tests could not be collected; (iii) samples were clotted or hemolyzed; (iv) subjects did not have an urgent or emergent blood culture during their intensive care unit stay.

geometric mean of fluorescence, were also not different across age groups (Figure $2 \mathbf{b}$ ).

\section{Neutrophil CD64 Expression}

At enrollment, the percentage of CD64+ neutrophils was different across age groups $(P=0.047)$ with term neonates $<7 \mathrm{~d}$ of age having higher percentages vs. 1-11-mo-old infants $(P=$ 0.004; Figure 2c). For CD16+ neutrophils, there was a trend toward a difference across age groups $(P=0.06$; Figure $2 \mathrm{~d})$. Trend testing demonstrated an inverse relationship between age group and percentage of $\mathrm{CD} 16+$ neutrophils positive for CD64 (Spearman -0.40 , regression $P=0.008$; Figure 2d).

The number of CD64 molecules per neutrophil ranged from 300 to 35,900 with no difference between age groups $(P=0.11$, Figure 2e). Among CD16+ neutrophils, there was a difference across age groups $(P=0.01)$, with preterm or term neonates $<7$ $\mathrm{d}$ of age having a higher number of CD64 molecules per neutrophil vs. 1-11-mo-old infants (both $P \leq 0.007$; Figure 2f). Trend testing demonstrated an inverse relationship between age group and number of CD64 molecules per CD16+ neutrophil with lower levels in older infants (Spearman -0.52 , regression $P<0.001$; Figure 2f).

\section{Biomarker Levels in Suspected Infection Samples}

Twenty-six subjects had study samples collected in association with a clinical evaluation for infection later in their intensive care unit (ICU) stay (suspected infection sample; Figure 1b). The majority of subjects were preterm ( $<36 \mathrm{wk}$ gestational age; Table 1). Ten (38\%) of these subjects, mostly from the 7-29 d and 1-11-mo-old age groups, were determined to be infected.
Twelve subjects had been given antibiotics within $5 \mathrm{~d}$ before collection of their suspected infection samples.

Individual leukocyte counts were measured in all suspected infection samples (Supplementary Figure S2 online). Only lymphocyte counts differed in infected vs. noninfected subjects $(P=0.04$; Supplementary Figure S2c online).

\section{Monocyte HLA-DR Expression}

Infected subjects had a lower percentage of HLA-DR+ monocytes than noninfected subjects $(P=0.02$, Figure 3a; AUC 0.78 , cut-off $99.5 \%$, sensitivity (Sn) 67\%, specificity (Sp) 63\%). There was no significant difference between infected and noninfected subjects in cell surface levels of monocyte HLA-DR expression (Figure $3 b$ ).

\section{Neutrophil CD64 Expression}

Infected subjects had a higher percentage of CD64+ neutrophils vs. noninfected subjects $(P=0.009$, Figure $3 c$; AUC 0.81 , cut-off $50.7 \%$, Sn $70 \%$, Sp $69 \%$ ). This difference was also seen for CD16+ neutrophils $(P=0.02$, Figure 3d; AUC 0.79, cutoff $67.5 \%$, Sn 67\%, Sp 69\%). Infected subjects had a higher mean number of CD64 molecules per neutrophil vs. noninfected subjects ( $P=0.04$, Figure 3e; AUC 0.75 , cut-off 2759 , Sn $60 \%, \mathrm{Sp} 63 \%)$ and a higher mean number of CD64 molecules per CD16+ neutrophil ( $P=0.04$, Figure 3f; AUC 0.76, cut-off 3146, Sn 67\%, Sp 64\%).

\section{Plasma CRP and PCT Levels}

CRP levels were available for 7 infected and 13 noninfected subjects. Infected subjects had higher CRP levels vs. noninfected 
subjects (median (IQR); 54.6 (8.7, 151.6) vs. 2.9 (2.9, 2.9); $P$ $=0.004$ ). Fifteen subjects (six infected; nine noninfected) had plasma collected for PCT analysis. Infected subjects had a trend toward higher PCT levels vs. noninfected subjects $(0.5$ $(0.3-1.1)$ vs. $0.3(0.1-0.5) ; P=0.09)$.

\section{Comparison of Enrollment and Suspected Infection Samples}

Eight study subjects had both enrollment and suspected infection study samples analyzed in this study (Supplementary Figure S3 online). Three of these eight subjects had an infection at the time of their subsequent infection work-up. The first subject demonstrated an increase in monocyte HLA-DR expression and a decrease in neutrophil CD64 expression. This subject was treated with antibiotics prior to collection of their suspected infection sample (started $24 \mathrm{~h}$ prior). The second subject showed an increase in both monocyte HLA-DR and neutrophil CD64 expression and the third subject showed a decrease in percentage of HLA-DR+ monocytes and neutrophil CD64 expression. Neither of these subjects received prior antibiotic treatment.

\section{DISCUSSION}

The objective of this study was to examine monocyte HLA-DR and neutrophil CD64 expression in relation to postnatal age and infection in critically ill infants. Using our enrollment samples, we found an age-related decrease in neutrophil CD64 expression but no significant age-related changes in monocyte HLA-DR expression. Using our suspected infection samples, we found a decrease in monocyte HLA-DR expression and an increase in neutrophil CD64 expression in infected vs. noninfected subjects.

Previous work has shown age-related changes in the levels of monocyte HLA-DR and neutrophil CD64 expression in children. Initial studies of neonatal monocyte HLA-DR levels found that neonates have lower levels of expression than adults $(24,25)$. However, our study is the first to examine agerelated monocyte HLA-DR expression in both neonates and infants. We found high levels of HLA-DR expression in infants of all ages with no significant differences between preterm and term neonates and older infants (Figure 2a,b). Earlier studies have also suggested age-related changes in neutrophil CD64 expression in preterm and term neonates (26). We found an age-related decrease in neutrophil CD64 expression among critically ill neonates and infants (Figure 2c-f). The levels of neutrophil CD64 expression we observed in preterm and term neonates were substantially higher than previously reported in healthy preterm and term infants (median 55-65\% CD64+ neutrophils vs. median 20\%) (26).

Previous reports have demonstrated an association between HLA-DR+ monocytes, CD64+ neutrophils, and infection in neonates. Our findings concur with others that have reported lower percentages of HLA-DR+ monocytes in infected vs. noninfected neonates (Figure 3a) $(24,25,27)$. The clinical utility of assessing HLA-DR expression on monocytes in diagnosing infection remains controversial (27). Others have focused on the prognostic value of monocyte HLA-DR expression
Table 1 Demographic characteristics of study subjects with enrollment and suspected infection study samples

\begin{tabular}{|c|c|c|}
\hline \multicolumn{3}{|l|}{ Enrollment samples } \\
\hline & No infection & Infection \\
\hline$N$ & 47 & $\mathrm{~N} / \mathrm{A}$ \\
\hline$\%$ male & 68 & $\mathrm{~N} / \mathrm{A}$ \\
\hline \multicolumn{3}{|l|}{ Age group } \\
\hline$<7$ d preterm $^{\mathrm{a}}$ & 20 & N/A \\
\hline$<7 \mathrm{~d}$ term & 13 & $\mathrm{~N} / \mathrm{A}$ \\
\hline $7-29 d^{b}$ & 8 & $\mathrm{~N} / \mathrm{A}$ \\
\hline $1-11 \mathrm{mo}^{\mathrm{c}}$ & 6 & $\mathrm{~N} / \mathrm{A}$ \\
\hline $\begin{array}{l}\text { Received antenatal steroids } \\
\text { (preterm only) }\end{array}$ & $18 / 31(58 \%)$ & $\mathrm{N} / \mathrm{A}$ \\
\hline \multicolumn{3}{|l|}{ Suspected infection samples } \\
\hline & No infection & Infection $^{d}$ \\
\hline$N$ & 16 & 10 \\
\hline$\%$ male & 62.5 & 40 \\
\hline \multicolumn{3}{|l|}{ Age group } \\
\hline$<7$ d preterm & 0 & 0 \\
\hline$<7 \mathrm{~d}$ term & 1 & 1 \\
\hline $7-29 d^{e}$ & 4 & 5 \\
\hline $1-11 \mathrm{mo}^{\mathrm{f}}$ & 11 & 4 \\
\hline $\begin{array}{l}\text { Received antenatal steroids } \\
\text { (preterm only) }\end{array}$ & $7 / 11(64 \%)^{9}$ & $5 / 8(63 \%)$ \\
\hline Prior antibiotic treatment & 7 & 5 \\
\hline Time since last dose $(\mathrm{h})^{\mathrm{h}}$ & $12.7(1.8-96.0)$ & $3.9(0.9-15.6)$ \\
\hline Duration of treatment $(\mathrm{d})^{\mathrm{h}}$ & $7.1(1.2-37.7)$ & $0.8(0.3-2.2)$ \\
\hline
\end{tabular}

aFifteen subjects $28-32$ wk gestational age; five subjects 33-35 wk gestational age. bSeven subjects were preterm (two subjects <28 wk gestational age; four subjects 28-32 wk gestational age; one subject 33-35 wk gestational age). Four subjects were preterm (two subjects $<28$ wk gestational age; two subjects 28-32 wk gestational

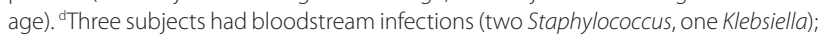
three subjects had pneumonia (one Escherichia, two Klebsiella/Staphylococcus); three subjects had necrotizing enterocolitis; one had outside diagnosis of bloodstream

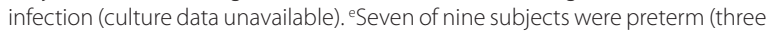
subjects $<28$ wk gestational age; four subjects $28-32$ wk gestational age). Thirteen of 15 subjects were preterm (five subjects < 28 wk gestational age; six subjects $28-32$ wk gestational age; two subjects 33-35 wk gestational age). ${ }^{9}$ One subject did not have information available and so was excluded from this ratio. ${ }^{~}$ Median (range).

largely in adult patients $(21,28-30)$. In one study of neonates with infection, those who survived had higher percentages of HLA-DR+ monocytes than those who died (31). We were unable to examine any prognostic significance of monocyte HLA-DR expression due to sample size restrictions.

Two meta-analyses of the use of neutrophil CD64 expression in diagnosing infection in infants and children found a sensitivity of $71-78 \%$ and a specificity of $81-87 \%(10,18)$. Our study involves only critically ill infants and showed lower performance characteristics (Figure 3). A previous study reported that a cut-off of 30\% CD64+ neutrophils was 97\% specific for infection in critically ill preterm and term neonates (11). In our study, a cut-off for CD64+ neutrophils of 50\% (all neutrophils; cut-off $50.7 \%$, AUC 0.81 , Sn $70 \%$, Sp $69 \%$ ) or $67 \%$ (CD16+ neutrophils; cut-off 67.5\%, AUC 0.79, Sn 67\%, Sp 
a

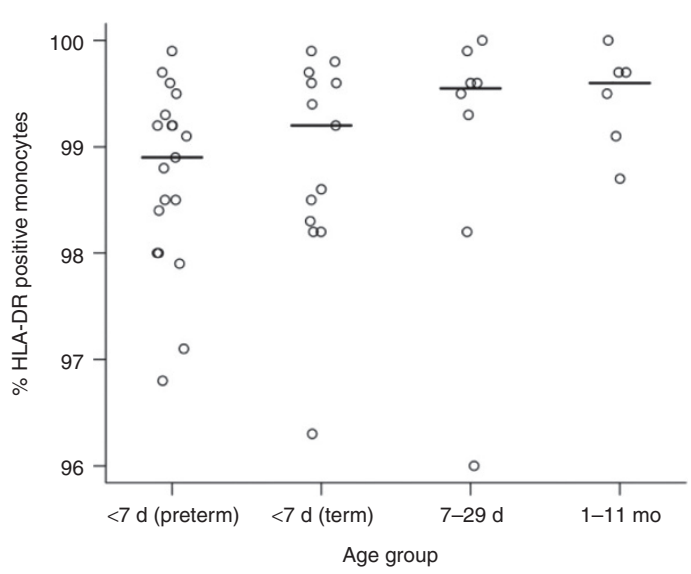

C
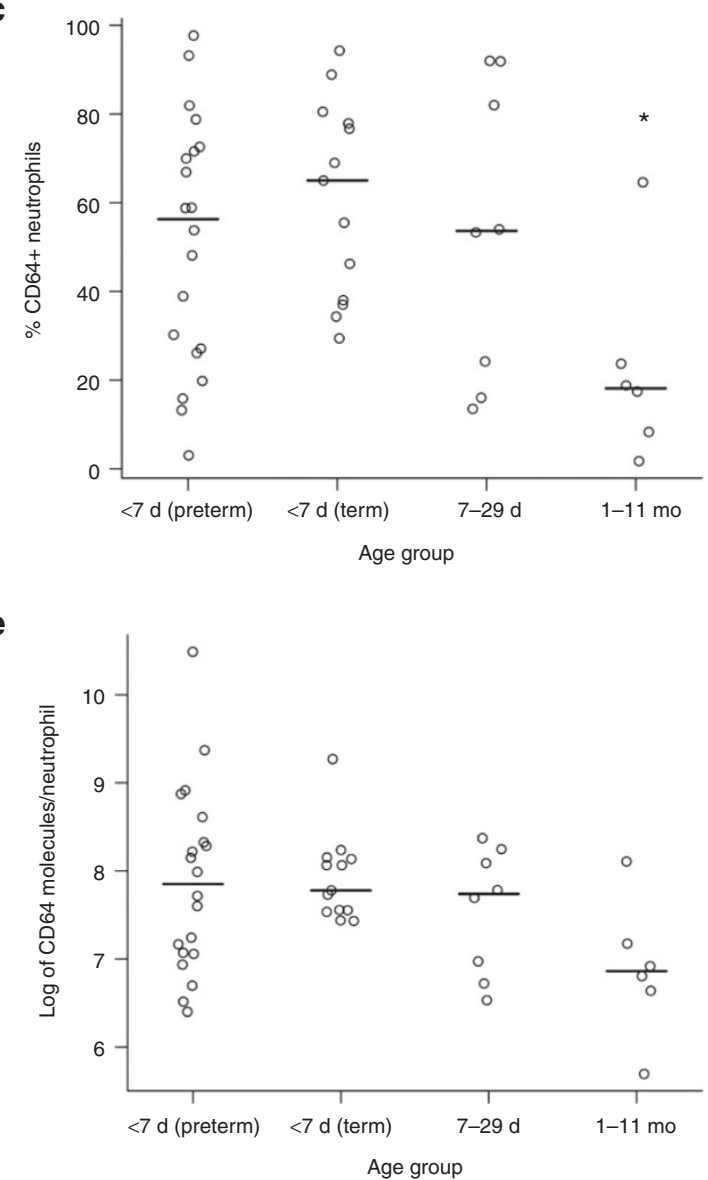

b

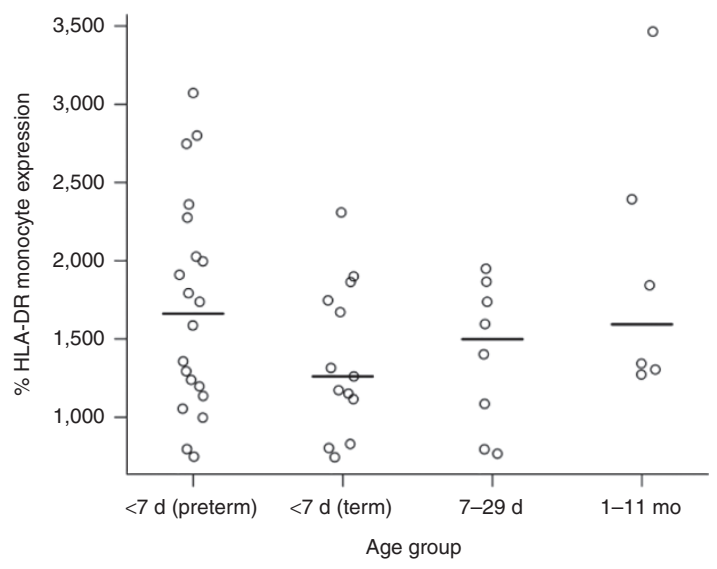

d

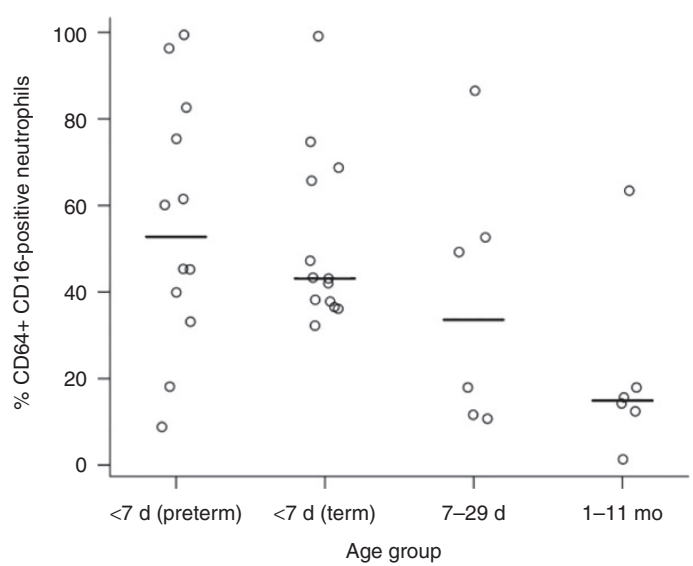

f

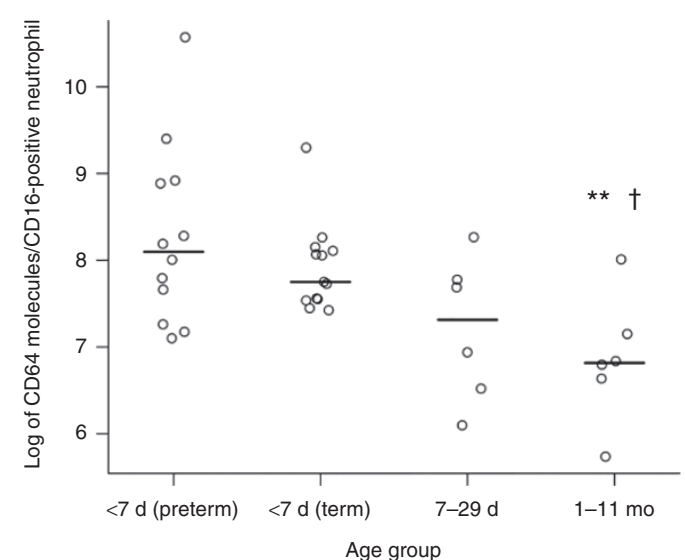

Figure 2. Cell surface marker levels at enrollment. Percentage HLA-DR+ monocytes (a), HLA-DR expression level on monocytes (b), percentage CD64+ neutrophils (c), percentage CD64+ among CD16+ neutrophils (d), natural logarithm of number of surface CD64 molecules per neutrophil (e), and per CD16+ neutrophil (f) at enrollment stratified by age group. CD16 analysis could not be performed on 10 enrollment samples. The horizontal line represents the median value among subjects in that age group. ${ }^{*} P<0.004 \mathrm{vs}$. term $<7 \mathrm{~d}$ olds; ${ }^{* *} P \leq 0.007 \mathrm{vs}$. preterm $<7 \mathrm{~d}$ olds; ${ }^{\dagger} p \leq 0.007 \mathrm{vs}$. term $<7 \mathrm{~d}$ olds.

69\%) provides the best balance of sensitivity and specificity (Figure 3c,d).

The strength of this study is that we also quantitated cellsurface levels of neutrophil CD64 molecules. We found increased levels of neutrophil CD64 expression in infected vs. noninfected infants (Figure 3e,f). Several reports have also found that neutrophil CD64 surface expression is higher in infected vs. noninfected infants (12-15,32). Some groups have advocated the use of a neutrophil CD64 index (patient's neutrophil CD64 mean fluorescence intensity compared to a commercially available standard) as a reproducible way to measure cell surface expression $(13-15,17,20)$. We used the Quantibrite bead system to convert mean fluorescence intensity to a number of surface molecules/cell similar to the work of $\mathrm{Ng}$ et al. and Du et al $(12,19,32)$. To detect infection, one proposed cutoff has been 4,000 and 6,136 CD64 molecules/neutrophil in 
a

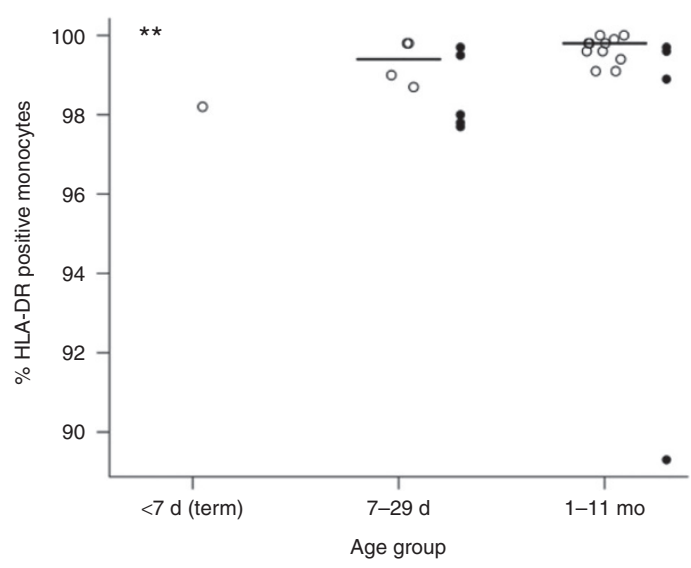

C

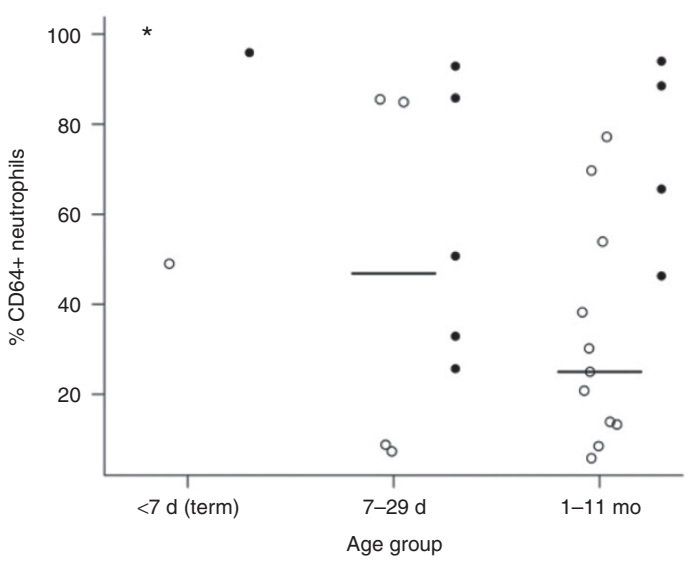

e

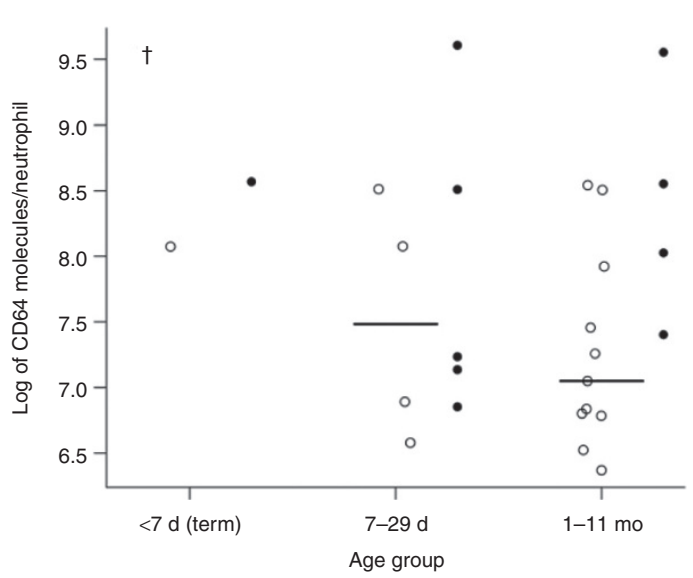

b

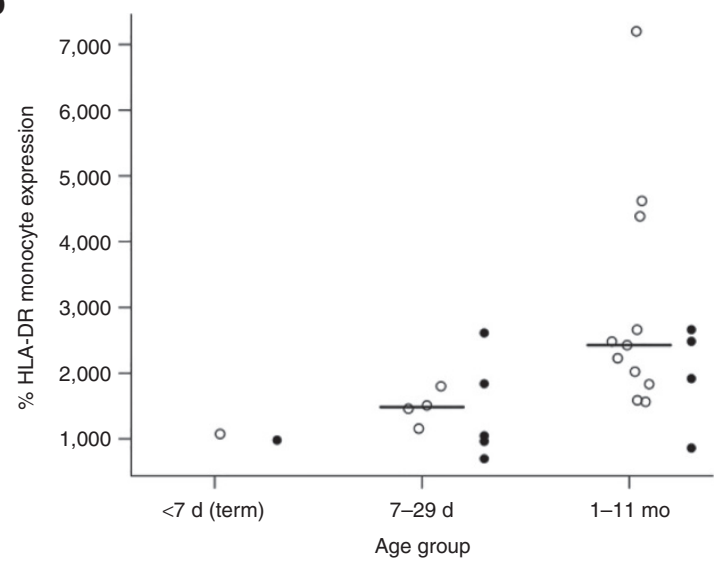

d

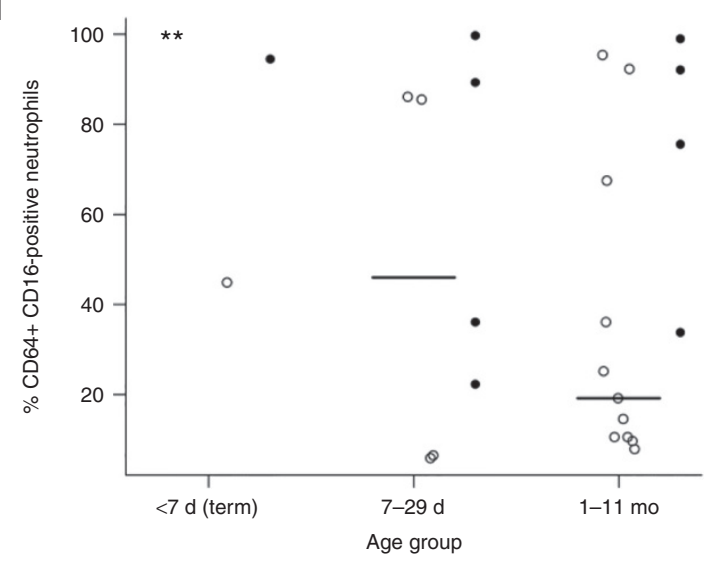

f

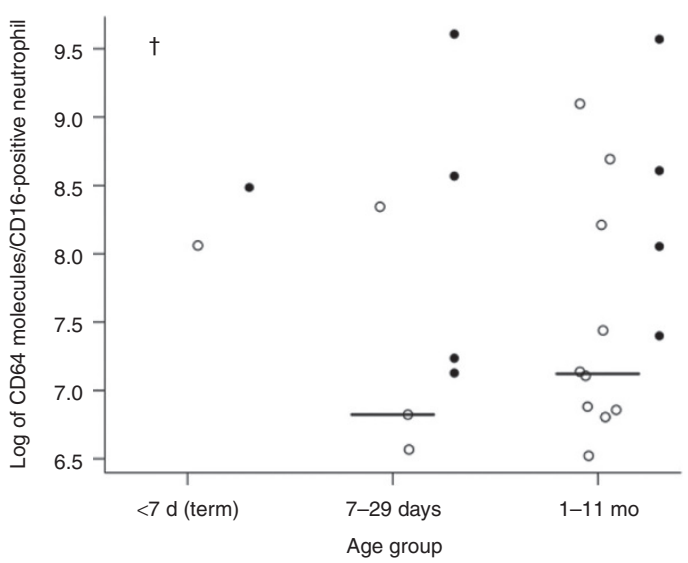

Figure 3. Cell surface marker levels at time of suspected infection. Percentage HLA-DR+ monocytes (a), HLA-DR expression level on monocytes (b), percentage CD64+ neutrophils (c), percentage CD64+ among CD16+ neutrophils (d), natural logarithm of number of surface CD64 molecules per neutrophil (e), and per CD16+ neutrophil (f) at time of suspected infection stratified by age group. White dots represent subjects without infection and black dots represent subjects with infection. The horizontal line represents the median value among subjects without infection in that age group. ${ }^{*} P=0.009$ for infected vs. noninfected subjects; ${ }^{* *} P=0.02$ for infected vs. noninfected subjects; ${ }^{\dagger} P=0.04$ for infected vs. noninfected subjects.

preterm and term neonates, respectively, both with a specificity nearing $90 \%(12,32)$. The other proposed cutoff advocated a much lower threshold (1,010 molecules/cell) for early-onset infection in preterm neonates. In our suspected infection samples, a cut-off of 2759 CD64 molecules/cell gives the best balance of sensitivity and specificity (Figure 3e,f; AUC 0.75 , Sn $60 \%$, Sp 63\%).
A potential confounding factor influencing our study's performance characteristics is that nearly half of our subjects received antibiotics within $5 \mathrm{~d}$ of their suspected infection samples (Table 1). In adults with localized musculoskeletal infections, prior antibiotic treatment was associated with a loss of neutrophil CD64 expression and subsequently lower sensitivity for detecting infection (33). A similar phenomenon 
could be occurring in our population who often receive antimicrobials as part of their clinical care.

Given the potential interactions between age, antibiotics, infection, and cell surface marker levels, our study design did allow the opportunity to analyze monocyte HLA-DR and neutrophil CD64 expression both at enrollment and later in the setting of suspected infection (Supplementary Figure S3 online). The number of subjects analyzed in this manner was small and no clear trends were observed with subsequent development of an infection. Given likely age- and antibiotic-related decreases in neutrophil CD64 expression, it may be difficult to then detect an increase in neutrophil CD64 expression due to infection. Neutrophil CD64 testing at the time of suspected infection could be of diagnostic value if age-related cut-offs between noninfected (normal) and infected (abnormal) subjects can be established and adjusted for recent antibiotic usage.

The study was limited by the fact that, for ethical reasons, we only collected blood samples for research if blood was being collected for other clinically indicated reasons. Since children who did not have blood collected for clinically indicated reasons were likely to be less severely ill, this may have enriched collection from the most critically ill subjects and thus those at the highest infection risk. Furthermore, small sample size limited the study's power and ability to examine marker performance adjusted for either age group or recent antibiotic treatment.

In summary, noninfected critically ill neonates and infants have an age-related decrease in neutrophil CD64 expression. Subjects with infection had lower monocyte HLA-DR expression and higher neutrophil CD64 expression than subjects without infection. However, further study is needed to establish normal and abnormal monocyte HLA-DR and neutrophil CD64 expression levels by age as well as presence/absence of recent antibiotic treatment.

\section{METHODS}

\section{Study Design and Patient Population}

This cross-sectional study was conducted within an institutional review board approved prospective cohort study of sepsis in the NICU and pediatric ICU (PICU) at Mayo Clinic Children's Center, Rochester, MN. Patients were eligible for the cohort study if they were hospitalized between 1 April 2009 and 30 April 2011. Members of the cohort were screened prospectively for eligibility for this cross-sectional study. The only inclusion criterion was an expected ICU length of stay of $3+$ days. The only exclusion criterion was an immunocompromised state, whether due to an underlying condition associated with decreased immune function (e.g., primary immunodeficiency) or receipt of immunosuppressive therapy (e.g., cancer chemotherapy, transplant immunosuppressive agents, biologic immune-modulating agents, corticosteroids except antenatal steroids for prematurity) during the prior $3 \mathrm{mo}$. Written informed consent was obtained from parents or guardians of eligible patients.

\section{Sample Collection and Processing}

Methods for study sample collection and processing in this population have been described previously (23). In brief, an initial sample could be collected from subjects if they had a planned blood draw for other clinical purposes within $72 \mathrm{~h}$ of enrollment and if they were $>1 \mathrm{~kg}$ in weight at the time of collection (enrollment sample). Samples also could be collected from subjects later in their ICU stay during clinical evaluation for a suspected new infection, as represented by collection of a blood culture as part of a clinically-indicated evaluation for suspected infection, unless another blood culture had been collected within the previous $7 \mathrm{~d}$ (suspected infection sample).

Samples consisted of heparinized whole blood $(0.5-1.0 \mathrm{ml})$, which was stored at $4{ }^{\circ} \mathrm{C}$ and analyzed by flow cytometry within $24 \mathrm{~h}$. If additional volume was available, an Ethylenediaminetetraacetic acid plasma sample $(0.25-0.5 \mathrm{ml})$ was collected and stored at $-80{ }^{\circ} \mathrm{C}$ for the analysis of PCT levels after study completion.

\section{Infection Classification and Antibiotic Assessment}

The electronic medical records of subjects were reviewed independently by two investigators (J.E.J., W.C.H.) using a standardized protocol to determine infection (e.g., bloodstream infection, urinary tract infection, pneumonia, necrotizing enterocolitis) within $3 \mathrm{~d}$ of any study sample collection. Investigators were blinded to flow cytometry, CRP, and PCT results. Bloodstream infection was determined using the laboratory-confirmed bloodstream infection definition from the Centers for Disease Control and Prevention's National Healthcare Safety Network (34). Pneumonia was defined as a chest radiograph with a new infiltrate, consolidation, or opacity described in the radiologist's report, tracheal secretions containing moderate to many white blood cells on Gram stain, and a positive tracheal secretion culture. Urinary tract infection was defined as a urine culture with $\geq 10,000$ colony-forming units of bacteria or yeast per $\mathrm{ml}$ or urinalysis demonstrating pyuria and positive Gram stain. Necrotizing enterocolitis was defined as a radiographic diagnosis of pneumatosis intestinalis or intestinal perforation and findings consistent with intestinal ischemia on surgical exploration of the abdomen. For a subject transferred from another healthcare facility, a diagnosis of infection made by an attending physician could satisfy our study definition even if sufficient clinical information was not available because the attending physician may have had access to additional relevant information from the referring facility. Study samples designations (infected or noninfected) were compared and discrepancies were resolved by consensus after joint review of the medical record.

Antibiotic administration records were reviewed for all study subjects that had a suspected infection sample collected given the potential effect of antibiotic usage on monocyte HLA-DR and neutrophil CD64 expression previously reported (33). Any antibiotics given within $5 \mathrm{~d}$ prior to suspected infection sample collection were recorded and both the time since most recent antibiotic administration and duration of antibiotic treatment prior to sample collection were calculated.

\section{Flow Cytometry Measurements and Analysis}

Heparinized whole blood samples were split in two aliquots for flow cytometry analysis. The first aliquot was stained using CD45 PerCP (BD Biosciences, San Jose, CA), CD163 APC (R\&D Systems, Minneapolis, MN), CD13 PE-Cy7 (BD Biosciences), HLA-DR APC-Cy7 (BD Biosciences), and CD14 PacificBlue (Invitrogen, San Diego, CA) antibodies, lysed using FACS Lysing Solution (BD Biosciences) and analyzed on a FACS Canto II flow cytometer (BD Biosciences) using FACSDiva software version 6.1 (BD Biosciences). The monocyte population was selected using side scatter, CD45, CD13, and CD14 gating. Monocyte HLA-DR expression was then quantified in two ways: (i) percentage of HLA-DR+ monocytes; (ii) level of monocyte HLA-DR expression (geometric mean of fluorescence).

The second aliquot of whole blood was stained with CD15 FITC (BD Biosciences), CD64 QuantibritePE/CD45 PerCP (BD Biosciences), CD13 PE-Cy7 (BD Biosciences), CD16 APC-Cy7 (BD Biosciences), and CD14 Pacific Blue (Invitrogen) antibodies and lysed using FACS Lysing Solution (BD Biosciences). Quantibrite PE beads (BD Biosciences) were run on a FACS Canto II flow cytometer (BD Biosciences) using FACSDiva software version 6.1 (BD Biosciences) to create a standard curve (surface molecules/cell) for quantitative CD64 analysis. For stained samples, the neutrophil population was selected using side scatter, CD45, CD13, and CD15 gating. CD64 and CD16 expression levels were then quantified on neutrophils. Neutrophil CD64 expression levels were assessed in the following two ways: (i) percentage of CD64+ neutrophils; (ii) number of CD64 molecules/ cell. CD16 analysis could not be performed on 10 enrollment samples. 


\section{Plasma CRP and PCT Measurement and Analysis}

For subjects who had suspected infection samples collected, an investigator abstracted plasma CRP levels measured within $3 \mathrm{~d}$ of study sample collection. Results reported as $<3 \mathrm{mg} / \mathrm{l}$ were recorded as $2.9 \mathrm{mg} / \mathrm{l}$. We also assessed PCT levels in additionally collected EDTA plasma using a commercially available BRAHMS Kryptor sensitive procalcitonin kit (Thermo Fisher Scientific LLC, Middletown, VA). Results reported as $<0.1 \mathrm{ng} / \mathrm{ml}$ were recorded as $0.09 \mathrm{ng} / \mathrm{ml}$.

\section{Statistical Analysis}

Study data were collected and managed using REDCap electronic data capture tools hosted at Mayo Clinic. REDCap (Research Electronic Data Capture) is a secure, web-based application (http://project-redcap.org/) designed to support data capture for research studies, providing: (i) an intuitive interface for validated data entry; (ii) audit trails for tracking data manipulation and export procedures; (iii) automated export procedures for seamless data downloads to common statistical packages; and (iv) procedures for importing data from external sources.

Subjects were stratified into three age groups $(<7 \mathrm{~d}, 7-29 \mathrm{~d}, 1-11$ mo) at the time of enrollment (demographic data), time of enrollment sample collection (enrollment sample analysis), and time of suspected infection sample collection (suspected infection analysis) since subjects changed age groups during the study. Subjects were subcategorized as preterm and term (preterm, $<36 \mathrm{wk}$ gestational age; term, $\geq 36$ wk gestational age). Study samples were assigned a designation of infection or no infection. Scatter plots and descriptive statistics were generated for each biomarker stratified by age group at the time of collection. In situations of highly skewed data, the log transformation was used for illustration purposes.

For enrollment samples from noninfected subjects, cell counts and cell surface marker levels were compared across four age groups using the Kruskal-Wallis test. Subsequent Wilcoxon rank sum pairwise testing was then performed for each significant Kruskal-Wallis test. To examine any trend between CD64 expression on CD16-positive neutrophils and age, we estimated the Spearman correlation coefficient and performed linear regression with the ranked data. Leukocyte counts, CRP, PCT, monocyte HLA-DR, and neutrophil CD64 expression levels in suspected infection samples were compared between infected and noninfected subjects using Wilcoxon rank sum tests. ROC curves were constructed and both AUCs and cut-offs for the best balance of sensitivity and specificity were calculated. For suspected infection samples, no adjustment for age group or recent antibiotic treatment was made due to sample size constraints. $P$ values $<0.05$ were considered statistically significant and $P$ values $<0.1$ were considered a trend toward significance. A Bonferroni correction was used in pairwise Wilcoxon rank sum tests to account for multiple testing; after correction, $P$ values $<0.0083$ were considered statistically significant. Only $P$ values that met significance or trend toward significance are reported. All analyses were performed using SAS version 9.

\section{SUPPLEMENTARY MATERIAL}

Supplementary material is linked to the online version of the paper at http://www.nature.com/pr

\section{ACKNOWLEDGMENTS}

We thank the staff of Mayo Clinic's Mobile Clinical Research Unit and the Mayo Clinic Medical Scientist Training Program for their support and resources. We would also like to thank faculty and staff of the NICU and pediatric ICU at Mayo Clinic Children's Center for all of their assistance with this study.

\section{STATEMENT OF FINANCIAL SUPPORT}

This work was supported in part by grants from the National Institute of Diabetes and Digestive and Kidney Diseases (Bethesda, MD; F30DK084671), the National Center for Advancing Translational Sciences (Bethesda, MD; UL1TR000135), and Mayo Clinic's Department of Pediatric and Adolescent Medicine (Rochester, MN). There are no potential conflicts of interest or financial disclosures.

\section{REFERENCES}

1. Kung HC, Hoyert DL, Xu J, Murphy SL. Deaths: final data for 2005. Natl Vital Stat Rep 2008;56:1-120.
2. Watson RS, Carcillo JA, Linde-Zwirble WT, Clermont G, Lidicker J, Angus DC. The epidemiology of severe sepsis in children in the United States. Am J Respir Crit Care Med 2003;167:695-701.

3. Connell TG, Rele M, Cowley D, Buttery JP, Curtis N. How reliable is a negative blood culture result? Volume of blood submitted for culture in routine practice in a children's hospital. Pediatrics 2007;119:891-6.

4. Polin RA; Committee on Fetus and Newborn. Management of neonates with suspected or proven early-onset bacterial sepsis. Pediatrics 2012;129:1006-15.

5. Newman TB, Puopolo KM, Wi S, Draper D, Escobar GJ. Interpreting complete blood counts soon after birth in newborns at risk for sepsis. Pediatrics 2010;126:903-9.

6. Da Silva O, Ohlsson A, Kenyon C. Accuracy of leukocyte indices and C-reactive protein for diagnosis of neonatal sepsis: a critical review. Pediatr Infect Dis J 1995;14:362-6.

7. Pourcyrous M, Bada HS, Korones SB, Baselski V, Wong SP. Significance of serial C-reactive protein responses in neonatal infection and other disorders. Pediatrics 1993;92:431-5.

8. Benitz WE, Han MY, Madan A, Ramachandra P. Serial serum C-reactive protein levels in the diagnosis of neonatal infection. Pediatrics 1998;102:E41.

9. van Rossum AM, Wulkan RW, Oudesluys-Murphy AM. Procalcitonin as an early marker of infection in neonates and children. Lancet Infect Dis 2004;4:620-30.

10. Cid J, Aguinaco R, Sánchez R, García-Pardo G, Llorente A. Neutrophil CD64 expression as marker of bacterial infection: a systematic review and meta-analysis. J Infect 2010;60:313-9.

11. Layseca-Espinosa E, Pérez-González LF, Torres-Montes A, et al. Expression of CD64 as a potential marker of neonatal sepsis. Pediatr Allergy Immunol 2002;13:319-27.

12. Ng PC, Li G, Chui KM, et al. Neutrophil CD64 is a sensitive diagnostic marker for early-onset neonatal infection. Pediatr Res 2004;56:796-803.

13. Bhandari V, Wang C, Rinder C, Rinder H. Hematologic profile of sepsis in neonates: neutrophil CD64 as a diagnostic marker. Pediatrics 2008;121:129-34.

14. Groselj-Grenc M, Ihan A, Derganc M. Neutrophil and monocyte CD64 and CD163 expression in critically ill neonates and children with sepsis: comparison of fluorescence intensities and calculated indexes. Mediators Inflamm 2008;2008:202646.

15. Icardi M, Erickson Y, Kilborn S, Stewart B, Grief B, Scharnweber G. CD64 index provides simple and predictive testing for detection and monitoring of sepsis and bacterial infection in hospital patients. J Clin Microbiol 2009;47:3914-9.

16. Jalava-Karvinen P, Hohenthal U, Laitinen I, et al. Simultaneous quantitative analysis of Fc gamma RI (CD64) and CR1 (CD35) on neutrophils in distinguishing between bacterial infections, viral infections, and inflammatory diseases. Clin Immunol 2009;133:314-23.

17. Streimish I, Bizzarro M, Northrup V, et al. Neutrophil CD64 as a diagnostic marker in neonatal sepsis. Pediatr Infect Dis J 2012;31:777-81.

18. Jia LQ, Shen YC, Hu QJ, et al. Diagnostic accuracy of neutrophil CD64 expression in neonatal infection: a meta-analysis. J Int Med Res 2013;41:934-43.

19. Du J, Li L, Dou Y, Li P, Chen R, Liu H. Diagnostic utility of neutrophil CD64 as a marker for early-onset sepsis in preterm neonates. PLoS One 2014;9:e102647.

20. Lynema S, Marmer D, Hall ES, Meinzen-Derr J, Kingma PS. Neutrophil CD64 as a diagnostic marker of sepsis: impact on neonatal care. Am J Perinatol 2015;32:331-6.

21. Spittler A, Roth E. Is monocyte HLA-DR expression predictive for clinical outcome in sepsis? Intensive Care Med 2003;29:1211-2.

22. Le Tulzo Y, Pangault C, Amiot L, et al. Monocyte human leukocyte antigen-DR transcriptional downregulation by cortisol during septic shock. Am J Respir Crit Care Med 2004;169:1144-51.

23. Juskewitch JE, Enders FT, Abraham RS, Huskins WC. Novel infrastructure for sepsis biomarker research in critically ill neonates and children. Clin Transl Sci 2013;6:21-5.

24. Kanakoudi-Tsakalidou F, Debonera F, Drossou-Agakidou V, et al. Flow cytometric measurement of HLA-DR expression on circulating monocytes in healthy and sick neonates using monocyte negative selection. Clin Exp Immunol 2001;123:402-7. 


\section{Articles $\mid$ Juskewitch et al.}

25. Skrzeczyñska J, Kobylarz K, Hartwich Z, Zembala M, Pryjma J. $\mathrm{CD} 14+\mathrm{CD} 16+$ monocytes in the course of sepsis in neonates and small children: monitoring and functional studies. Scand J Immunol 2002;55:629-38.

26. Fjaertoft G, Håkansson L, Foucard T, Ewald U, Venge P. CD64 (Fcgamma receptor I) cell surface expression on maturing neutrophils from preterm and term newborn infants. Acta Paediatr 2005;94:295-302.

27. Ng PC, Li G, Chui KM, et al. Quantitative measurement of monocyte HLA-DR expression in the identification of early-onset neonatal infection. Biol Neonate 2006;89:75-81.

28. Lekkou A, Karakantza M, Mouzaki A, Kalfarentzos F, Gogos CA. Cytokine production and monocyte HLA-DR expression as predictors of outcome for patients with community-acquired severe infections. Clin Diagn Lab Immunol 2004;11:161-7.

29. Oczenski W, Krenn H, Jilch R, et al. HLA-DR as a marker for increased risk for systemic inflammation and septic complications after cardiac surgery. Intensive Care Med 2003;29:1253-7.
30. Gomez HG, Gonzalez SM, Londoño JM, et al. Immunological characterization of compensatory anti-inflammatory response syndrome in patients with severe sepsis: a longitudinal study ${ }^{*}$. Crit Care Med 2014;42:771-80.

31. Genel F, Atlihan F, Ozsu E, Ozbek E. Monocyte HLA-DR expression as predictor of poor outcome in neonates with late onset neonatal sepsis. J Infect 2010;60:224-8.

32. Ng PC, Li K, Wong RP, Chui KM, Wong E, Fok TF. Neutrophil CD64 expression: a sensitive diagnostic marker for late-onset nosocomial infection in very low birthweight infants. Pediatr Res 2002;51: 296-303.

33. Tanaka S, Nishino J, Matsui T, Komiya A, Nishimura K, Tohma S. Neutrophil CD64 expression in the diagnosis of local musculoskeletal infection and the impact of antibiotics. J Bone Joint Surg Br 2009;91: $1237-42$.

34. Horan TC, Andrus M, Dudeck MA. CDC/NHSN surveillance definition of health care-associated infection and criteria for specific types of infections in the acute care setting. Am J Infect Control 2008;36:309-32. 